\title{
Validación de un taller para el manejo del estrés en personal de enfermería Validating a stress-management workshop for nursing staff
}

\author{
Heidi G. Redondo-Martínez ${ }^{a}$, Lorenzo Martínez-Hernández ${ }^{a}$, Alfredo Hernández-Morales ${ }^{a}$, \\ Yerarldi L. Méndez-Hernández ${ }^{a}$, Fernando Ochoa-Cortés ${ }^{b}$
}

\begin{abstract}
:
In order to avoid economic loses and worker productivity, most companies and institutions implement actions to reduce work relatedstress. However, some institutions do not take into account the effect of stress on employee productivity and service quality. Psychological abuse directed to inpatients, is a worldwide issue. Some reasons are health personnel overwork, deficient organization, etc. We propose to implement a stress-management workshop in health institutes and determine its validity. Using stress-assessment tools, we will evaluate stress level in nursing staff allocated to inpatient care areas, due to their greater interaction with the vulnerable population. After taking this workshop, we expect nursing staff will perform their duties appropriately and thus contribute to customer satisfaction in response to the provided service.
\end{abstract}

Keywords:

Workshop for managing work stress, Work stress and Psychological abuse of the patient

\section{Resumen:}

La mayoría de las empresas e instituciones, realizan actividades para reducir el estrés laboral para no afectar sus inversiones y productividad. Sin embargo, algunas instituciones no valoran cómo afecta el estrés laboral la productividad de los empleados o la calidad en el servicio. El maltrato psicológico a la persona hospitalizada es un problema que se presenta a nivel mundial. Algunas de sus causas son el exceso de trabajo, que el personal no sepa cómo organizarse, etc. Nosotros proponemos implementar un taller para el manejo del estrés y determinar su eficacia en las instituciones sanitarias. Utilizando instrumentos de evaluación para determinar si sufren de estrés, se evaluará al personal de enfermería del área de hospitalización, ya que tiene mayor contacto con población vulnerable. Después de tomar este taller se espera que el personal realice su trabajo de manera adecuada y que haga sentir al cliente satisfecho de los servicios brindados.

\section{Palabras Clave:}

Taller para el manejo del estrés, estrés laboral, maltrato psicológico al paciente 


\section{Taller para disminuir el stress}

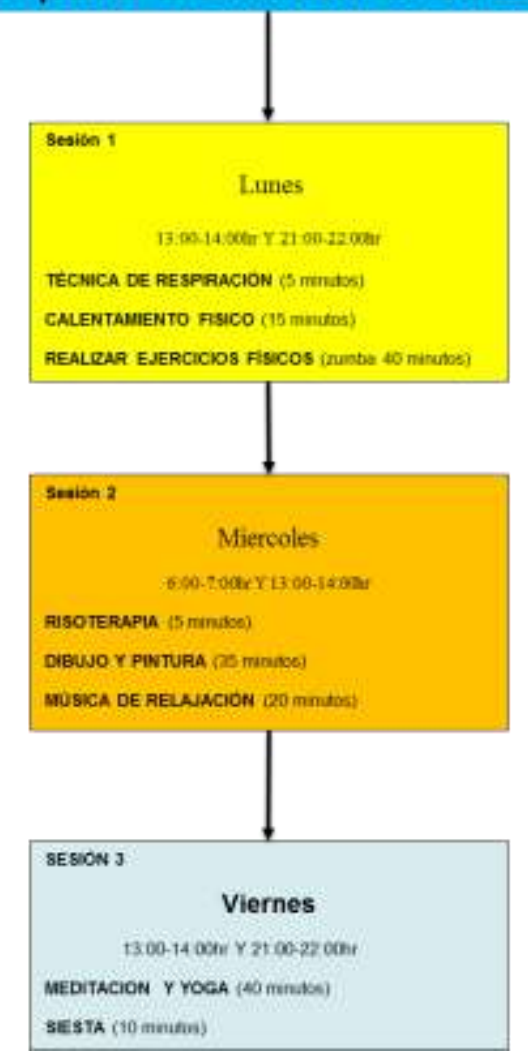

Una forma de reducir el estrés del personal de enfermería sería un taller que ayudaría a prevenir o reducir los factores y estresores relacionados con el aspecto laboral, obteniendo como resultado una mejor calidad de trabajo y tener un cliente satisfecho, tranquilo y cómodo. El mejoramiento del estado de salud lo más rápido posible y tener una enfermera capaz y con los requisitos necesarios para realizar procedimiento y cuidados como lo marcan las normas y los protocolos de la institución y de los manuales a seguir, esperando obtener por último a un cliente feliz. La carga psicosocial dentro y fuera de la institución en el personal de enfermería es acumulada y les impide realizar su trabajo de manera eficiente, de tal manera que, se plantea métodos y estrategias para elaborar talleres de relajación personal; actividades recreativas y físicas; así como terapia ocupacional para disminuir el estrés incluso para prevenir en lo futuro, el síndrome de "burnout".

\section{Referencias:}

(1) Del Hoyo Delgado, M. (2012). Estrés laboral. I. Seguridad e Higiene en el Trabajo, Ed. España, Madrid. Recuperado el 27 de 04 de 2019 , de http://www.insht.es/InshtWeb/Contenidos/Documentacion/FICHAS\%20DE\%20PUBLICACIONES/FONDO\%20HISTORICO/DOCUMENTOS\%20DI VULGATIVOS/DocDivulgativos/Fichero\%20pdf/Estres\%20laboral.pdf

(2) Selye, H. (1975). Tensión sin angustia. Guadarrama, España, Madrid.

(3) Silpak, O. (1991). Historia y concepto del estrés. Recuperado el 27 de 04 de 2019, de https://www.alcmeon.com.ar/1/3/a03_08.htm 